Journal Reference: International Journal of Current Trends in Science and Technology [Online ISSN: 0976-9730; Print ISSN: 0976- 9498; Section: Social Science], Vol. 8, Issue. 02, Page no: SS 2055720563 (Published in INDIA in the Year 2018)

\title{
Evolution through Cognitive Processes: \\ Implicative assertions for Knowledge Development and Social Changes
}

\author{
Biplab Chattopadhyay \\ Taki Government College, Taki, North 24 Parganas, Taki - 743429, West Bengal, India
}

\begin{abstract}
The process evolution is all pervading and influences everything in the material world. We explore as to how evolution shapes the course of knowledge development including innovations in science and technology. We examine the implicative role of evolution in determining the course of social changes and the plunges it eventually take. The fact that the cognitive processes, contradiction, giving vent to evolutionary proceed, and conflict and continuity, are allied to evolution, is emphasized. Relevance of views and concepts of natural sciences is examined for social dynamics and social changes.
\end{abstract}

\section{Embarking Overture:}

Nature flows with time and so does our society carrying along the accumulated load of human civilization for the sole purpose of achieving more and more homogeneity with the passage of time.

Coinage of the term homogeneity is from the sphere of science. Any system pertaining to our very own worldly nature, be it physical, chemical, biological, mathematical or similar variants, would always exhibit naturally lawful tendency to become homogeneous. Likewise, human society, by virtue of its being governed by natural laws, would also always pursue the path to homogeneity, and so it does. In case of human society, this pursuit to homogeneity is termed as socialization. In any sub-variant of nature, called a system, homogeneity signifies maximization of entropy $^{1}$ or the number of states accessible to the system, which, in turn, means freer and greatly uninhibited interactions among the constituents of the system. Similarly, homogeneity or the state of socialization within the human society implies greater freedom for its constituent humafolks in mutual interactions, uninhibited expression of views or thoughts, indiscriminate share in all social products and abandoned participation in social processes.

The pathway to added homogeneity or to a more socialized state, both of which are analogous, find their ever rolling upkeep in the process called evolution. Acquiring the state of more homogeneity, or, to that effect, the state of being more socialized, is precisely the purpose of evolution. Every system, whatsoever, belonging to the material nature of mundane world, always eyes to get into the more homogeneous or socialized build through the evolutionary track. The reason behind a system's affinity towards more homogeneity lies in its tendency or urge to be progressively more stable, and such stability is guaranteed by evolutionary proceedings under the aegis of Darwinian natural selection ${ }^{2}$. Here comes the cyclic rolling of three factual processes contradiction, conflict and continuity, occupying three intervening locations in the distinctive pathway of evolutionary course with the entire proceed being spread plausibly on the perimeter of a circle and signifying essentially the closed cycle trail of evolution.

Evolution in a biological system proceeds through some operational phases, prevalently numbered at three. In an apparently stable system, emergence of another distinctive daughter stream or emergence of two daughter streams negating the one parent is inevitable. This surfacing 
of two from one signifies Contradiction. Two contradicting streams, because of hard-core type repulsion, cannot occupy the same space at the same time and hence a fight for supremacy erupts expectantly which is demonstrative of the cognitive process Conflict. Resolution of conflict is an essential necessity and is a binding inflicted by nature which essentially upholds the constancy of its own (natural) abundance as well as the process of evolution. Consequently, nature does select the rather fit stream from the two simultaneous. The fittest ultimately survives and acquires stability in due course attesting Continuity or flow of the stream undergoing evolution. Actually, continuity of the best suited stream signifies completion of the perceived cyclic chain in evolution from where a new cycle may get started. Therefore to say, entities or streams of them, whatsoever, pertaining to nature, always pursue the repetitive cyclic path of evolution albeit with enhanced stature in each significant full cycle.

The process of acquiring knowledge to unfold mysteries of nature, that eventually enriches human civilization, too follows the path of evolution. Existing concepts or thoughts about any natural phenomenon or any characteristic manifestation of it, as a result of ever stretching interactions, give rise to parallel set of concepts or thoughts which would definitely be in unambiguous contradiction with the established parent stream of concepts/thoughts. Each set of thoughts, flowing parallel, strives to come to prevalence and thus conflicts between them come to fore. Further, in the prospective timeline, nature itself acts as the best judge and selects the most logical of set of thoughts to live on and get established in due course. A specific stream of thoughts or concepts, when established as appropriate (at particular space-time), becomes stable and hence continue to maintain its stability until it undergoes the next cycle of evolution. This factual espousing of evolutionary track by concepts or thoughts or knowledge and their eventual transmutation through refinement, is indicative enough that knowledge development does take place through evolutionary corridor and consequently the state of human civilization finds progressive elevation through each cycle of knowledge evolution.

Actually, development of knowledge through evolutionary trail is a necessity for progressive ascend of human civilization and this very inevitable act leads to acquisition of added insights about natural phenomena. Added insights thence generate fresh innovations inflicting renewed endeavours in all the fields of knowledge including science and technology. Thereupon, newer directions in the practice of science become observable and technological knowhow finds legitimate progress by leaps. And, the sequential process of contradiction, conflict and continuity, embedded in the close-chain cyclic track of evolution, commands significant bearing in shaping directions of contemporary science and technology by virtue of knowledge development. Social processes or the society as a whole, owing to their unhindered allegiance to evolution, too follow the sequential evolutionary flow-path embedding the factual processes contradiction, conflict and continuity in order for their own improved shaping with time and thus strive to acquire greater stability by taking plunge into a more homogeneous state in each cycle of evolution. This adherence of the social realm to the three cognitive processes (con-words) in its own evolution, manifestly imply an unending relevance and unscratched significance of the cyclic rolling of these factual processes contradictions, conflicts and continuities for even the present-day understanding of knowledge development or knowledge growth.

In conforming to the views asserted so far, it becomes imperative on us to explore in clarifying details the interconnection of contradiction-conflict-continuity with the process of evolution and hence emphasize their bearings in the field of science \& technology and the society we live in. This is precisely the target and in the next few sections efforts will be steamed to achieve this goal.

Organization of the rest of the article is done as follows. In section-2, evolution in a biological system is considered with elaboration on the embedding of the cognitive processes contradiction, conflict and continuity. Relevance of these concepts for scientific and technological upgradation 
has been analyzed in section-3 in relation to the process of knowledge development. Section-4 includes exploration on ever changing characteristic of our society from the viewpoint of evolution. Herein, the dynamical track of social evolution is discussed including portrayal of social changes based on ideas and views from science. A summary of the write-up along with bottom-line clarifications is included in section-5. In the apparent winding-up efforts, some thoughts are scribbled in wrapping-remarks as section-6.

\section{The Cognitive Processes Contradiction, Conflict and Continuity in Evolution:}

To create an understanding about the connection of evolution with the cognitive processes, contradiction, conflict and continuity and to realize the significance of these processes in keeping up the dynamic stature of evolution, we need to look through the modalities of biological evolution in a definitive species. Let us consider that a stable species of established characteristic details is undergoing biological evolution. All the members of the definite species in consideration are exhaustively constituted by a huge but finite set of functionally coordinated organs. This is same as saying that a definite living entity is an integration of a set of finitely many organs where the more complex the species-members be, the number of functionally coordinated organs in the integrated set becomes proportionally increased ${ }^{3}$. All of these organs in a living entity are undoubtedly dynamic in the sense that they suffer growth, take part in various lively syntheses and thereby keep themselves working, maintaining dynamicity for well defined purposes of life. In course of continuous and purposeful acts of organs, such as growth or synthesis, departure from expected outcome may happen because of faults created in the basic building blocks of organs, apparently termed as genes. In stricter biological terminology, one would say that while performing growth or synthesis work, organs may find occurrence of unexpected change in the genetic code and a consequent genetic mutation ${ }^{4}$ in its structure and performance. Accruing mutation of a kind (or many kinds) in an aggregate of species-members gets magnified and exists alongside those having organs of no genetic mutation. In course of time, mutated organs would develop a will to supersede and thus negate the non-mutated organ content members and vice versa. Ultimately, the process called natural selection take precedence choosing the beneficial mutations to be preserved and passed on to the next generation. Over a long time-scale, beneficial mutations accumulate, magnify and result in entirely different organs from the original, thereby giving rise to a new species.



Figure-1: Illustrating the cyclic path of evolution, including its different stages, along with contradiction, conflict and continuity being embedded within.

Biological evolution of a new species from an old one, as narrated above, is mostly the Darwin's theory that revolves around the premise of natural selection where modern era concept of mutation from molecular biology is being infused in logical terms. In this whole process of biological evolution, the three con-words, signifying change-over points to different stages of evolution, are truly existent and extensively prevalent.

An organ of biological nature and defined characteristics, when gets the favour of nature and thus embark on an easy-going path, simultaneously becomes prone to random mutations in its building blocks, the genetic codes. Such mutation leads to a different organ having characteristics other than the parent one. Emergence of a characteristic new organ, disregarding the rules 
governing the parent organ, would, by all means, give vent to contradiction erupting between two streams or lines. Further, in the time line, coexisting two different organs, governed by two separate bundles of rules, would try to negate each other for acquiring eminence and thus would be in serious conflict. The process of natural selection would step in here to resolve the conflict between the two and the organ having beneficial characteristics, would be permitted to sustain as well as pass on their characteristics to the descending generations illustrating continuance or continuity of beneficial mutation. The whole process of biological evolution can, thus, be picturised as three phases spread in a circle, as depicted in Figure-1, with contradiction, conflict and continuity denoting the three interphasing points (or areas) on the cyclic chain. Time flows clockwise along the cycle starting from the point of continuity, visiting phases of evolution embedding contradiction and conflict in-between and then coming to continuity again completing the specific cycle of evolution. A cyclic picture of evolutionary track signifies that evolution is unstoppable and progresses with time. Further, specific general phases of evolution are sequenced in closed chain that repeats with time in consecutive cycles with contradiction-conflict-continuity as demarcations exemplifying their importance in the upkeep of evolution.

\section{Evolution in Science and Technological Innovations:}

The ever developing stance of science and the consequent enhancement of technological knowhow ritually follow the path of evolution. Development in science means acquiring knowledge about material abundance of nature, both animate and inanimate, in terms of plausible logical parlance and derivation. And, accumulation of scientific knowledge inherently imply enhancement of technological expertise. In general, therefore, development of knowledge is at the helm of the development of science and technology, and, here comes the playing of evolution together with the three con-words. To explore the interconnections of knowledge development with evolution along with the con-words, let us do a couple of case studies.

From the time of Sir Isaac Newton, mechanical behaviour of material is understood within the ambit of Newtonian Mechanics ${ }^{\mathbf{5}}$ where time is considered to be absolute. Absoluteness of time claims that the time-gap between two consecutive events, as measured from different reference frames in motion relative to each other, would turn out to be the same. Counter experience to this absolute time concept had probably been there for long. However, Albert Einstein first emerged to contradict the concept of absolute time and presented logical arguments in support of his refute. The contradiction thus brought, took shape in due course into conflicting concepts about the characteristics of time-coordinate in mechanics. Resolution of the conflict was being entrusted on nature where time-perspective experimental evidences and deductive logical reasoning uphold the non-absolute character of time. Thus, the views put forth by Einstein sustained and so continued giving vent to a new branch of physics known as relativistic mechanics. However, it should be noted that Newtonian mechanics still remain in force as the initial stairs to reach the floor of relativistic mechanics which gain practical significance when relative velocity between frames is comparable to that of light.

Let us now drift to another example. During the course of scientific innovations, necessity of high magnetic field (strength) was felt at some point. Strength of magnetic field produced in an electromagnet could be increased by increasing electric current in the winding coil. However, inevitable finite resistance of winding wire, that generates heat with high current, have eventually been capping the strength of magnetic field. But, inevitability or resistance of a material, with the advent of superconductivity as evidenced by Kamerling Onnes's experiment on Mercury ${ }^{6}$, is contradicted and zero resistance of material (under certain conditions) became reality. Emergence of zero resistance state conflicted the established viewpoint of heat generation by electric current and the concept of un-resisted super-current flow came to exist, persisted and continued. Here, 
development of knowledge through the natural evolutionary track enhanced the technological knowhow in terms of very high magnetic field production.

\section{Bearing of Natural Sciences in Social Dynamics:}

Social changes, like many other mundane phenomena, are attributed to evolution. Natural scientific concepts do have manifestation in social characteristics and they govern too the dynamics of the very society we live in. Society undergoes continuous evolution searching for a better position in terms of homogeneity and freedom of its constituents and thereby take plunge into the energy minimum state. This process of social change, local or global, always remains in force, uninhibited and unabated.

Passage of the society towards more homogeneous states could be understood in terms of a space time dependent state function (or free energy functional as in science) where the entity called society always endeavours to get into the local minima of the state function. In reference to the diagram in Figure-2, let us consider that society is sitting in the minima of the state function marked A at some specified space-time. Since there exists another minima B in the proximity, lower than A, the system society would naturally tend to move to this lower minima for acquiring more homogeneity and stability by virtue of minimizing free energy. A system's free energy $(F)$ bears the relationship ${ }^{7}$

$$
F=U-T S
$$

with its internal energy $U$, temperature $T$ and entropy $S$. Quite often, a system tries to minimize its free energy through maximization of entropy. Entropy $(S)$, perceived to signify the degree of disorder of a system, is expressed as ${ }^{1}$

$$
S=k \ln \Omega
$$

where $k$ is a physical constant named after Ludwig Boltzmann and $\Omega$ stands for the number of states accessible to the system. Thus, increase in $\Omega$ would lead to enhanced entropy $S$. The meaning attached to higher $\Omega$ is that the system becomes less constrained and can be in any of the large number of states. This very concept, when translated in social terms means that constituents of the more entropic state of society would have amplified choice to remain in any of the large number of states and hence would enjoy more freedom. Further, large $\Omega$ indicates that the system constituents get evenly spread in all available states which mean a more homogeneous attire of society and enhanced liberty for its constituents. Since the state at minimum is somewhat trapped, minor fluctuations won't be able to displace it and thus the society at minimum would become more stable. The arguments above clearly establish that entropy does also indicate the level of freedom and degree of homogeneity of the constituents of a system.

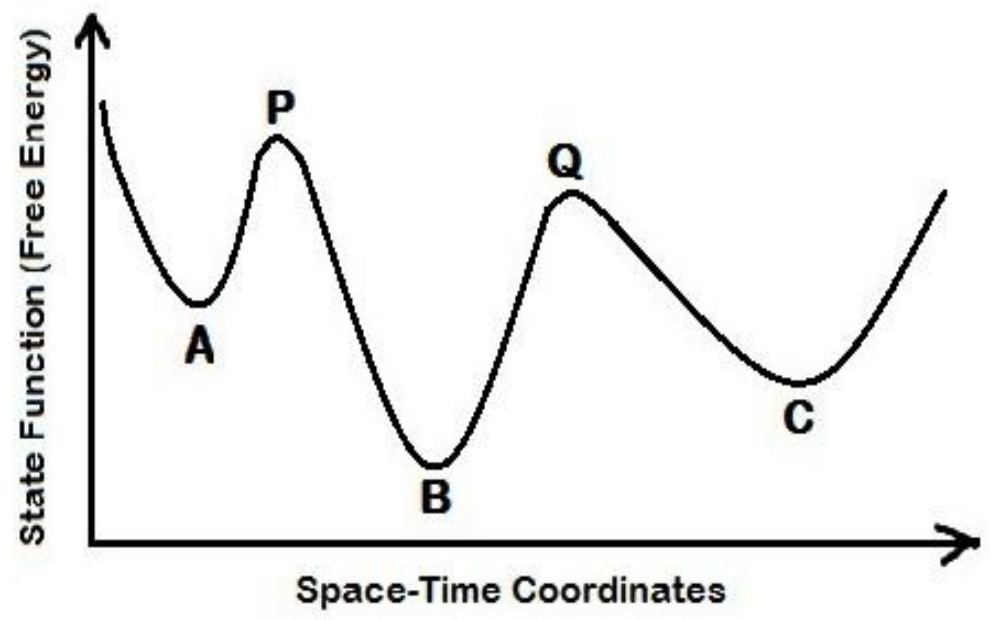

Figure-2: Diagrammatic representation of free-energy state-function with minima marked as A, B, C and up-hill points (maxima) denoted as $\mathrm{P}, \mathrm{Q}$

Passage of the system (society) from $\mathrm{A}$ to $\mathrm{B}$ must proceed through the uphill point $P$, which means an increase of the free energy. The system (society) increases its free energy (F) by virtue of increasing internal 
energy (U) achieved by enhanced internal fluctuations. Once the system (Society) acquires free energy as that of the point $\mathrm{P}$, it rolls down to lower minimum $\mathrm{B}$ immediately.

Narratives so far in this section emphasize the applicability of natural laws, views and concepts in case of society and thus endeavour to add knowledge to the understanding of social dynamics as well as changes occurring to the state of the society. Views, concepts and laws of nature that find application here, have got seasoned again and over through the evolutionary cycle embedding contradiction-conflict-continuity and have thus got established to date with unchallenged validity. However, applicability of the natural laws, in the fashion it is done here for society, would probably invite contradictions and naturally roll on assuming the cyclic path of evolution for continuous refinement and hence a better understanding would always emerge.

\section{Summarizing Bottom-line Clarifications:}

In this article, we have tried to explore the correspondence between the methodical course contradiction-conflict-continuity, and the path of evolution, in worldly aspects like mutagenic biological changes, knowledge development and social dynamics. We have shown that evolutionary changes do occur in closed cycle sequence of well defined mechanisms where the cognitive processes contradiction, conflict and continuity are steadfastly embedded in narrated order. Continuity in the evolutionary cycle signifies stability of the concerned change and it also marks the start of a new cycle of evolution through renewed contradictions. Some example studies are done in the fields of science and technology to show how contradictions to the contemporary concepts lead to newer directions and innovations.

We have also analyzed the dynamical attribute of society within the framework of natural laws highlighting the way society moves from one state to the other and its tendency to attain more homogeneity in every change through evolution. Consequent upon the fact that change is an unchanging feature, evolution, which inflicts change, is an inevitable process in this world and the universe, and contradicting the present is a must to ignite evolution so that hope for better future remains alive.

\section{Wrapping Remarks:}

As the saying goes, the process 'change' is unchangeable, so does every damn thing within the ambit of natural abundance. Quite evidently, thus, changes do always occur in society, evolving or placing it continually to the state of greater homogeneity with the definitive purpose to make it free from inequalities, disparities, divides and exploitations. At this point, the pertinent queries that could very well swamp our intellect must be raised and the same refers to the specificities of the role played by the constituents of society in its evolutionary plunge into ever enhanced homogeneous state. The emphasis prevalently lies on the sole question as to what proactive role the members of the species Homo Sapiens should play in the process of evolutionary changes of the Society in acquiring more homogeneity.

A plausible response to the posed quest is essential for making any headway in any of the intellectual or practical fields. To my perceived thoughts, apparently engagements of humanfolks in combating the very many avenues of exploitations existing in the society at its numerous spatial locations in innumerable forms, is the specific necessary role the human entities must play proactively at this age of ours. Combating or fighting against exploitations at locations could be thought of as nucleation of small ordered blobs (with associated homogeneity) in the ocean of an otherwise disordered state. With the passage of time and with simultaneous unabated fight against exploitation, ordered (homogeneous) blobs would grow in size and towards criticality (rising tempo of the fight against exploitation), as the correlation length gets larger, the ordered blobs 
would get connected to each other. At the critical point (peaked tempo of the fight against exploitation), the correlation length would have the infinite stretch and the infinite correlation length would make all the ordered blobs to get joined together leading to a completely ordered state thereby marking a first order phase transition ${ }^{8}$ in the system and the whole of the society would probably be the sea of perfect order with associated homogeneity.

\section{References:}

1. P. Attard, "Thermodynamics and Statistical Mechanics", Elsevier (2002).

2. K. A. Francis, "Charles Darwin and the Origin of Species", Greenwood Press, London (2007).

3. T. Audesirk and G. Audesrik, "Biology: Life on Earth", Prentice Hall, New Jersey, USA (1996).

4. A. J. F. Griffiths, W. M. Gelbart, J. H. Miller and R. C. Lewontin, "Modern Genetic Analysis", W. H. Freeman, New York (1999).

5. H. J. W. Muller-Kirsten, "Classical Mechanics and Relativity", World Scientific (2008).

6. D. van Delft, "Freezing Physics", Royal Netherlands Academy of Arts and Sciences (2007).

7. I. N. Levine, "Physical Chemistry", McGraw Hill: University of Brooklyn (1978).

8. G. Venkataraman, “The Many Phases of Matter”, University Press, India (1991). 